\title{
Numerical Comparison of the Method of Transport to a Standard Scheme
}

\author{
Tim Kröger ${ }^{\mathrm{a}, 1}$, Sebastian Noelle ${ }^{\mathrm{a}, 1}$ \\ ${ }^{a}$ RWTH Aachen, Institut für Geometrie und Praktische Mathematik, \\ Templergraben 55, D-52056 Aachen
}

\begin{abstract}
In previous joint work with Susanne Zimmermann, we derived Fey's Method of Transport (MoT), a multidimensional flux vector splitting scheme, from gas kinetic theory via quadrature. Now we present a number of numerical tests in one and two space dimensions showing that similarly to many other flux vector splitting and kinetic schemes, the MoT is very dissipative. In order to quantify its numerical dissipation, we compare the second order MoT-ICE [11] to the classical, very simple, and at the same time very robust Harten-Lax-van Leer (HLL) scheme (also in a second order version), whose most significant drawback is its comparatively high dissipativity. Our numerical experiments indicate that the MoT-ICE is approximately as dissipative as the much cheaper HLL scheme. Recall that the HLL scheme does not suffer from any of the known multidimensional instabilities, which have been a prime motivation for the development of Riemann solver free schemes.
\end{abstract}

Key words: multidimensional systems of hyperbolic conservation laws, Fey's Method of Transport (MoT), HLL scheme, numerical comparison $M S C$ 2000: 35L65, 76M12

Email addresses: kroeger@igpm.rwth-aachen.de (Tim Kröger), noelle@igpm.rwth-aachen.de (Sebastian Noelle).

1 Revised version, Jan.8, 2003. Submitted to Computers and Fluids.Supported by DFG-Priority Research Program "Analysis and Numerics for Conservation Laws" (ANumE) and EU-IHP Network "Hyperbolic and Kinetic Equations" (HYKE). 


\section{Contents}

1 Introduction 2

2 The governing equations 4

3 Choice of the test problems 4

4 Description of the schemes 5

$5 \quad$ Numerical results 5

5.1 Stationary contact 5

$\begin{array}{lll}5.2 & \text { Stationary disc } & 10\end{array}$

5.3 Oblique shear wave 12

$\begin{array}{lll}5.4 & \text { Strong stationary shocks } & 14\end{array}$

$\begin{array}{lll}\text { 5.5 Shock entropy wave interaction } & 20\end{array}$

6 Conclusion 20

References 23

\section{Introduction}

Many physical problems (for instance gas dynamics, magnetohydrodynamics, traffic flow) can be modelled by systems of hyperbolic conservation laws,

$$
\partial_{t} \boldsymbol{U}+\nabla_{\underline{x}} \cdot \underline{\boldsymbol{F}}(\boldsymbol{U})=0,
$$

if certain effects (for instance viscosity) are neglected. One of the first algorithms to compute solutions to conservation laws was introduced by Godunov [7] in 1959. His scheme is based on exactly solving a Riemann problem at each cell interface and then projecting the solution back onto the space of piecewise constant functions. An efficient variant of Godunov's method are the simplified "approximate" Riemann solvers introduced by Roe [17], Harten, Lax, van Leer [8], Osher et al [3,12], and others.

Due to the enormous complexity of the multidimensional Riemann problem, Godunov's approach cannot be applied directly to multidimensional systems. However, there are some standard techniques to extend his scheme to more space dimensions, e.g. dimensional splitting (on a cartesian mesh) or a finite 
volume framework (on an unstructured mesh). Piecewise polynomial reconstructions and a Runge-Kutta time step can be used to increase the formal order of accuracy. For a detailed description of Riemann solvers and Riemann solver based schemes see the textbook of Toro [23].

Despite the great success of these schemes, there is an ongoing discussion started by Phil Roe himself in 1986 [18] - whether one-dimensional Riemann solvers do justice to the multidimensional effects arising in such systems. In 1994, Quirk [16] presented a number of typical examples where many of the traditional Godunov-type schemes fail, unless they are stabilized by adding numerical viscosity. Riemann solver free schemes claim to handle multidimensional effects better, so that these problems are avoided.

The present article focusses on one of these Riemann solver free schemes, Fey's Method of Transport (briefly called MoT), derived in [4-6]. In previous work of the authors jointly with Susanne Zimmermann [10], we presented some new connections between several Riemann solver free schemes. One result was a new derivation, from gas kinetic theory, of those state and flux decompositions on which Fey's method is based. Now, we numerically compare the MoT in it's ICE version [11] to the Riemann solver of Harten, Lax, and van Leer [8] (briefly called HLL). The HLL scheme is known to be very simple and at the same time very robust but suffers from being rather dissipative: Contact discontinuities or, more generally, density variations are smeared out considerably. On the other hand, Quirk [16] already pointed out that the damping effect of the HLL scheme prevents it from producing multidimensional instabilities or unphysical solutions. Moreover, Pandolfi and D'Ambrosio [13] analyzed two of the instabilities Quirk had observed, namely the carbuncle phenomenon and the odd-even-decoupling (the latter also known as crossflow-instability). They came to the conclusion that a scheme which damps out density variations usually produces correct results for this type of test problems. In fact, they classify the HLL scheme as a 'carbuncle-free scheme'. Conversely, schemes which keep contacts sharp can be expected to suffer from multidimensional instabilities. This is, for example, the case for the HLLC scheme, a modification of HLL which preserves stationary contact discontinuities (see Toro, Spruce, Speares [24]). Pandolfi and D'Ambrosio classify the HLLC scheme as 'strongly carbuncle prone'.

Carrying this discussion one step further, we would like to propose that a multidimensional scheme that claims to be better than Riemann solver based schemes should firstly be less dissipative than HLL, and at the same time it should be carbuncle-free. The numerical results presented below, however, show that the MoT does not meet the first requirement. In fact, it is at least as dissipative as HLL.

The diffusivity of the MoT-ICE did not become apparent in [11] because there 
Noelle only treated the shallow water equations, which do not contain the linear field present in the Euler equations. We first realized this excessive dissipation in the dissertation of von Törne [22], where for one Euler test calculation, MoT-ICE was compared with LeVeque's CLAWPACK. However, such a comparison did not seem to be completely fair, because CLAWPACK is based on Roe's Riemann solver which automatically preserves stationary contacts. This eventually lead us to the present comparison of MoT-ICE and HLL.

We would like to add a word of caution: our second order version of HLL failed to converge for an oblique shear-discontinuity, while MoT-ICE did fine. This needs further investigation.

\section{The governing equations}

In all numerical test problems in this paper, we consider the Euler equations of gas dynamics. These equations take the form (1.1) where

$$
\boldsymbol{U}=\left(\begin{array}{c}
\rho \\
\underline{m}^{\mathrm{T}} \\
E
\end{array}\right), \quad \underline{\boldsymbol{F}}(\boldsymbol{U})=\left(\begin{array}{c}
\underline{m} \\
\underline{m}^{\mathrm{T}} \underline{u}+p \underline{\mathbf{1}} \\
(E+p) \underline{u}
\end{array}\right),
$$

and $\underline{m}=\rho \underline{u}$ is the momentum. The system is closed by the equation of state for an ideal gas,

$$
E=\frac{p}{\gamma-1}+\frac{1}{2} \rho \underline{u}^{2}
$$

where $\gamma$ is the adiabatic quotient and has a value of 1.4, valid for air, unless stated differently.

\section{Choice of the test problems}

A major difficulty in computing solutions to hyperbolic systems of conservation laws is the sharp resolution of contact discontinuities. This is desirable in many applications (e.g. shear- and boundary layers in aerodynamics). Note that shock waves are inherently compressive, and most schemes can resolve them within a few grid cells. Contrary to that, there is no compressive mechanism for linear waves, and for many schemes the width of the computed contact discontinuities grows with time. This smearing is a good measure of 
the diffusivity of a given scheme. A few selected Riemann solvers (Godunov, Roe, Osher-Solomon) are able to keep a stationary contact sharp. Others, like HLL, smear stationary contacts considerably, and much work has been invested to improve the HLL solver in this respect (see Einfeldt's HLLEM [2] and Toro et al.'s HLLC [23,24]). Note that moving contacts are smeared by any scheme due to the cell averaging at the end of each time step.

Thus our numerical experiments start with stationary contacts. In particular, we compute planar contacts aligned with, and oblique to, the cartesian grid, a circular contact, and oblique shear waves. We also include examples of strong stationary shocks and a shock entropy wave interaction. We choose the standard HLL solver for comparison because it is one of the most robust, but also most diffusive Riemann solvers. We believe that it is fair to demand that a newly developed multidimensional high resolution scheme should be less diffusive than HLL.

\section{Description of the schemes}

The MoT-ICE is implemented according to [11], using Fey's discrete flux decomposition $[6,(18)-(21)]$ which we derived from gas kinetic theory in $[10$, Example 2.7]. Data are reconstructed by piecewise linear functions, seperately in each spatial direction, using the monotonized centered slope limiter (MinMod2) in each component of the flux decomposition and conservative variables.

For comparison we use Einfeldt's [2] HLLE version of the Harten-Lax-van Leer scheme, raising it to second order accuracy via piecewise linear reconstructions in space and Heun's Runge-Kutta timestep.

All computations use a regular cartesian grid and CFL number 0.45.

\section{$5 \quad$ Numerical results}

\subsection{Stationary contact}

We start with a very simple problem: A stationary one-dimensional Riemann problem. We set $p=1, u=0$ and $\rho=\rho_{\mathrm{l}}=3$ (for $x<0$ ) or $\rho=\rho_{\mathrm{r}}=1$ (for $x>0$ ). The exact solution obviously remains constant for all time. We computed this problem with $\Delta x=1 / 256$ and a computational domain which is large enough such that any effects originating at the contact at $x=0$ are 


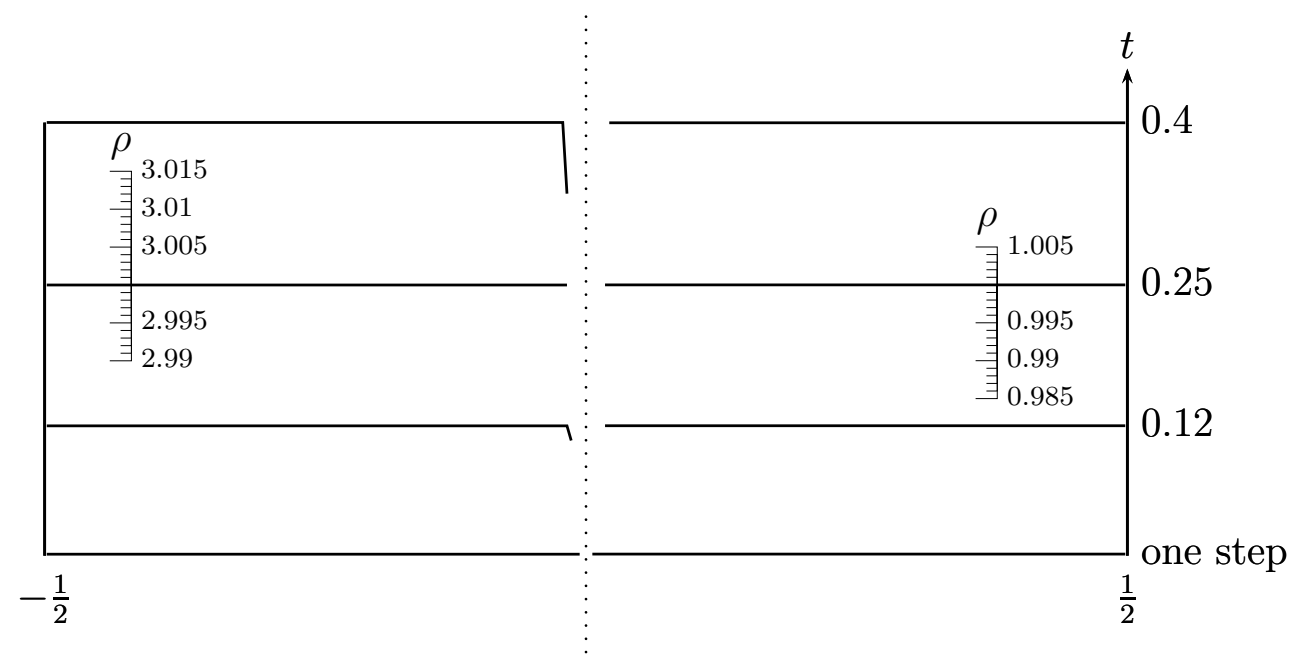

Fig. 5.1. $\rho$ component of the stationary contact problem, using second order HLL. Plot is split at the discontinuity - note the different scales to the left and right. Solution is shown at times $\Delta t, 0.12,0.25$ and 0.4 .

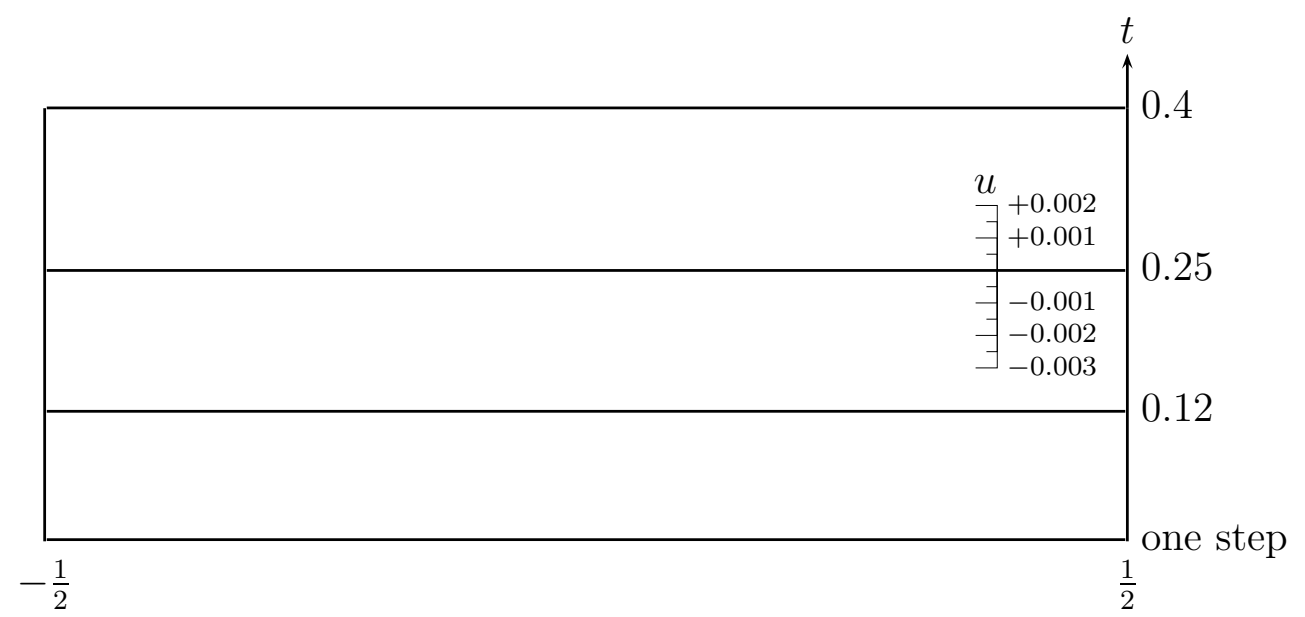

Fig. 5.2. $u$ component of the stationary contact problem, using second order HLL.

not able to reach the boundary within the time up to which the computation is carried out. (We show only the domain $[-0.5,0.5]$, but the real computational domain is larger.) This prevents effects which are due to the handling of the boundary conditions.

Results for this problem are shown in figures 5.1 to 5.3, where we first used the HLL scheme. Each plot displays the respective component at various selected time levels.

We see that the HLL scheme conserves the $u$ and $p$ components exactly while the $\rho$ component is smeared out quite heavily. (The $\rho$ plot is a split plot where each side is cut off at the position where the smearing begins. So the width of the gap between the two sides is a measure for the intensity of the smearing.) 


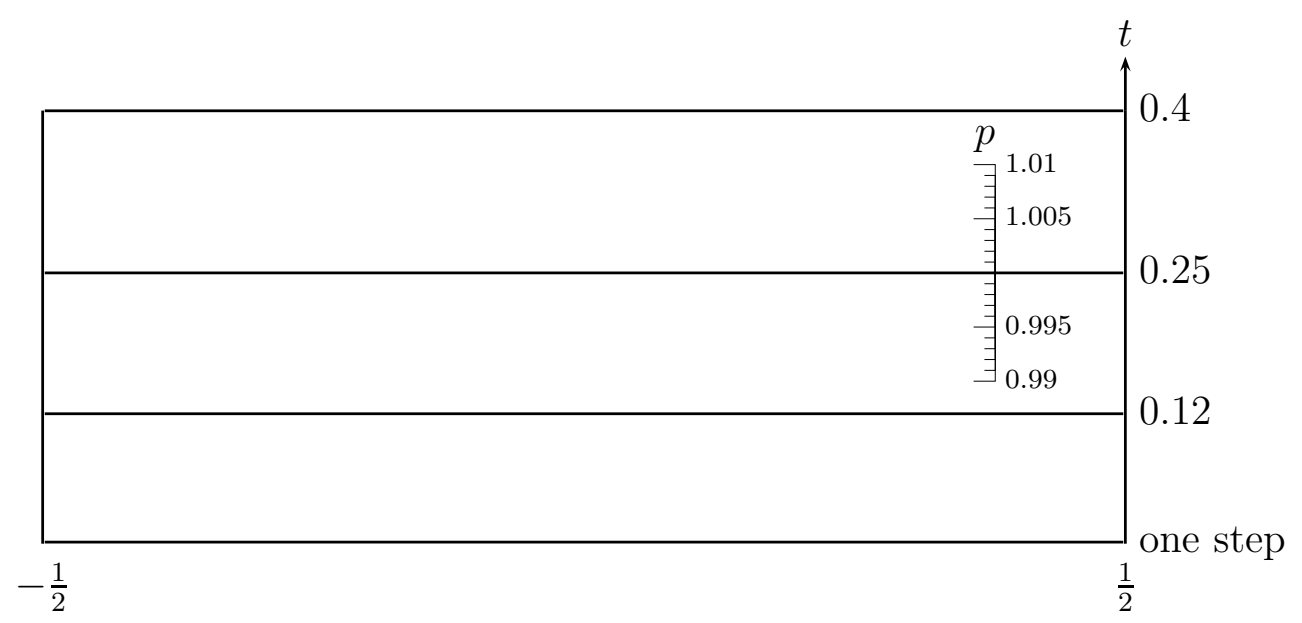

Fig. 5.3. $p$ component of the stationary contact problem, using second order HLL.

In fact, it is true, that the HLL scheme leaves pressure and velocity absolutely constant (up to machine precision) for all time (for an ideal gas) if they are constant in the initial data. This statement applies for first and second order versions, for arbitrarily many space dimensions, for any choice of the signal velocities and for any slope limiter out of the class of limiters that were discussed by Sweby [21]. The proof mainly consists in noting that

- the mapping from the primitive to the conservative variables,

$$
\left(\begin{array}{c}
\rho \\
\underline{u}^{\mathrm{T}} \\
p
\end{array}\right) \mapsto\left(\begin{array}{c}
\rho \\
\underline{m}^{\mathrm{T}} \\
E
\end{array}\right),
$$

is in the case of constant $\underline{u}$ and $p$ a first-degree polynomial of $\rho$ in each component, and thus, the difference of two neighboring states (in conservative variables) is a linear function of the difference of the $\rho$ values, and thus, the quotient of two differences is the same in all conservative components; and

- the flux matrix $\underline{\boldsymbol{F}}(\boldsymbol{U})$ can be written in the form

$$
\underline{\boldsymbol{F}}(\boldsymbol{U})=\boldsymbol{U} \underline{u}+\underline{\boldsymbol{Q}}(\underline{u}, p)
$$

where $\underline{\boldsymbol{Q}}(\underline{u}, p)$ is some matrix not depending on $\rho$ (which thus is constant in the considered case).

For details, see Kröger [9].

Figures 5.4 to 5.6 show the result of the same problem, but now using MoTICE. First, we see that there are now small waves in all components, originating at the contact and moving to both sides with the respective speed of sound. These perturbations arise in all three components, even in the first 


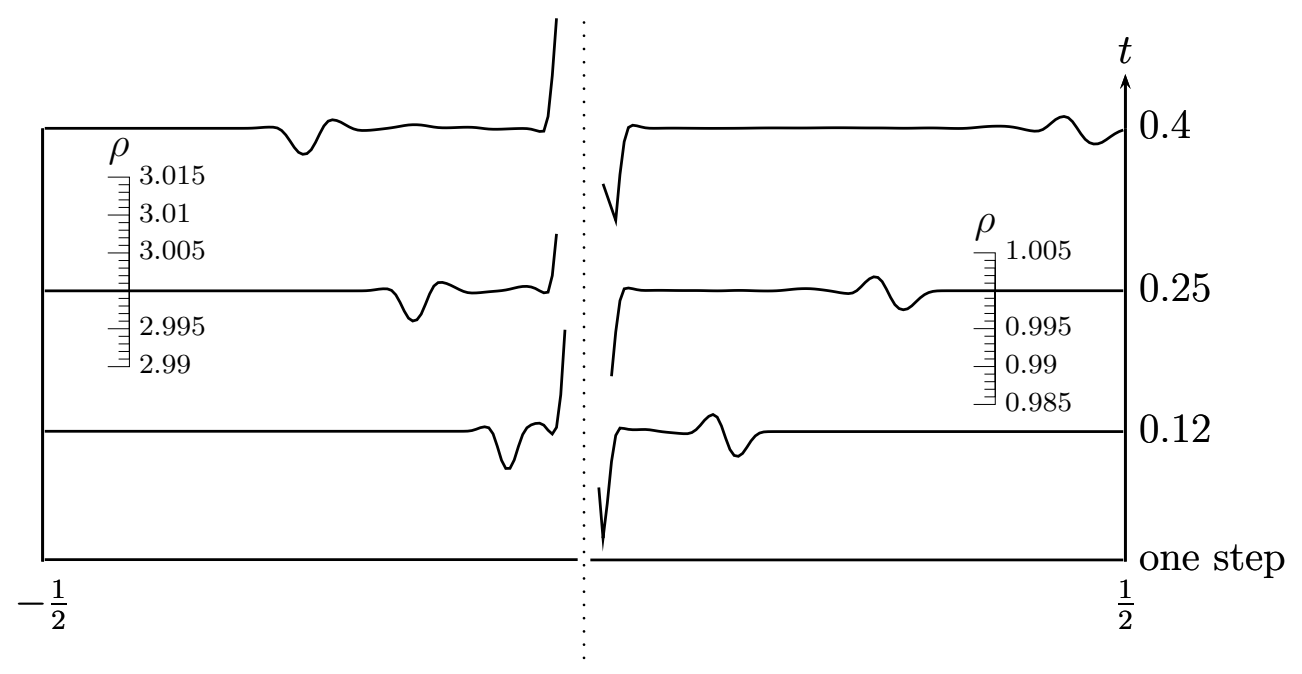

Fig. 5.4. $\rho$ component of the stationary contact problem, using second order MoT-ICE. Plot is split at the discontinuity - note the different scales to the left and right. Solution is shown at times $\Delta t, 0.12,0.25$ and 0.4 .

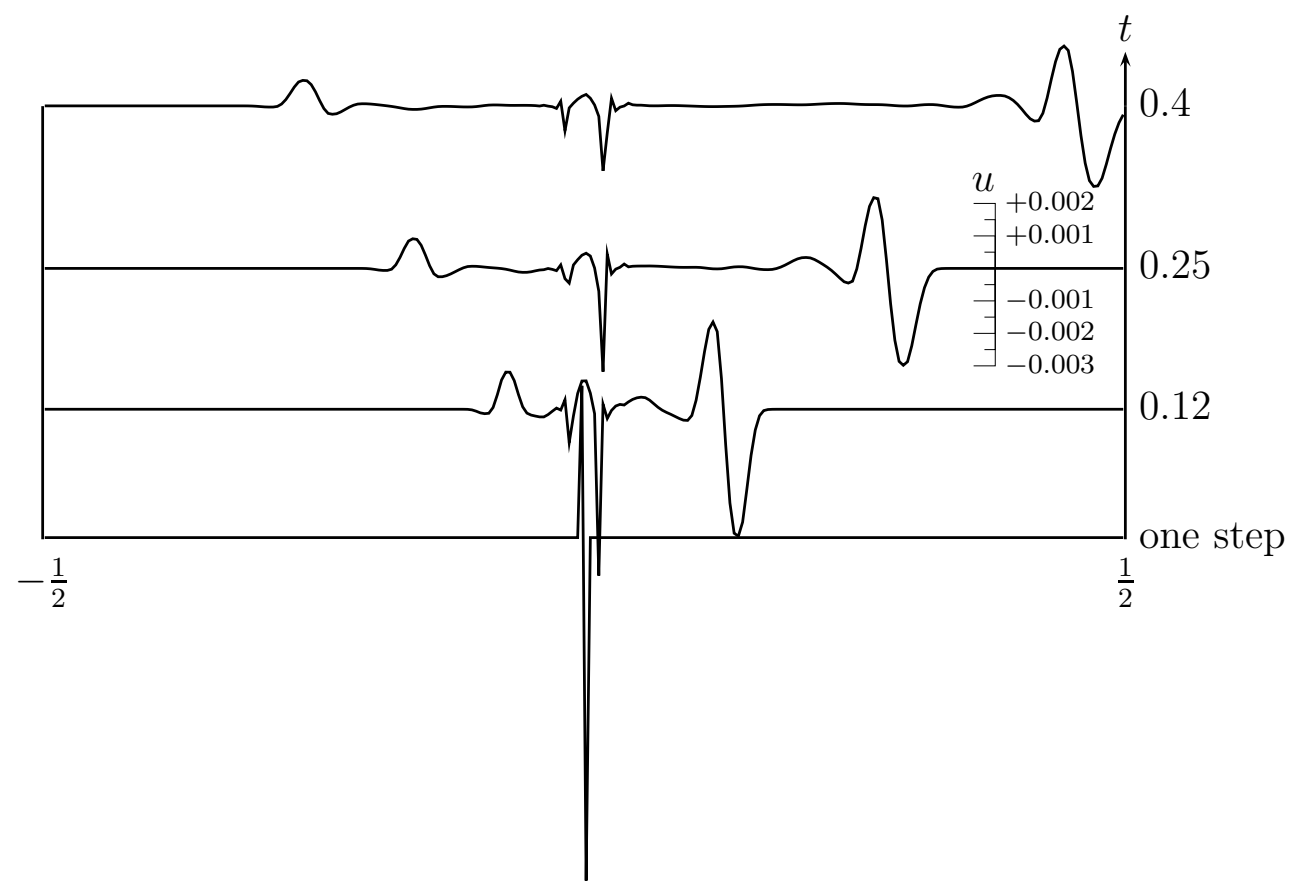

Fig. 5.5. $u$ component of the stationary contact problem, using second order MoT-ICE. 


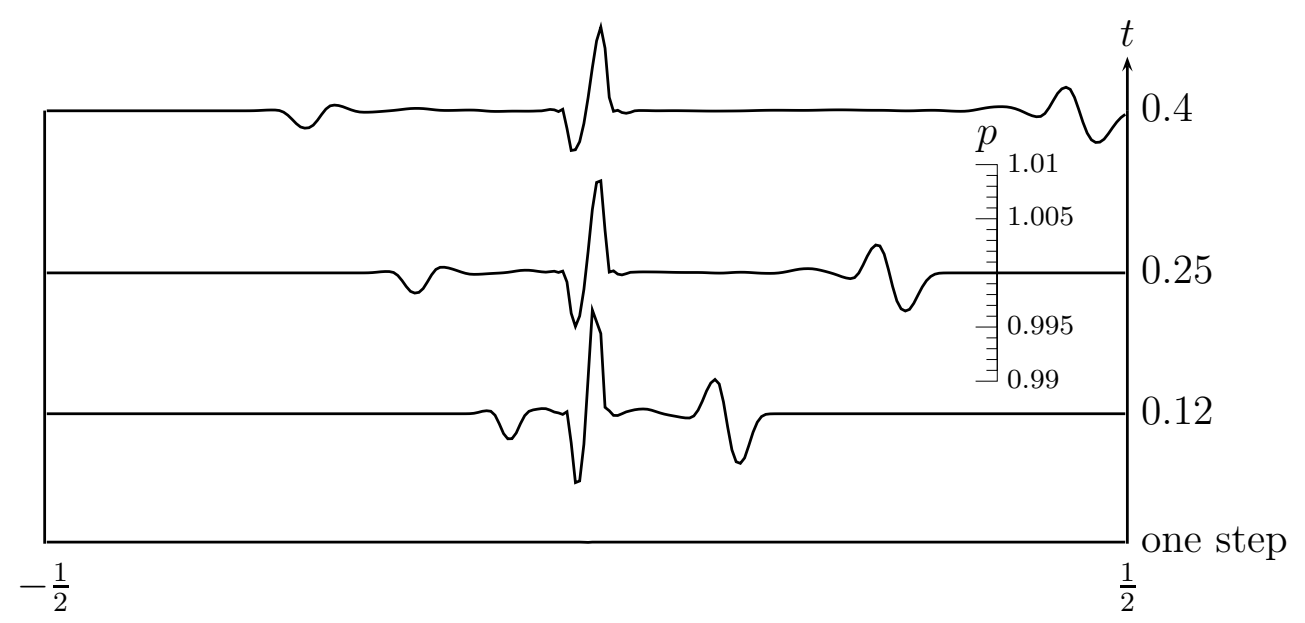

Fig. 5.6. $p$ component of the stationary contact problem, using second order MoT-ICE.

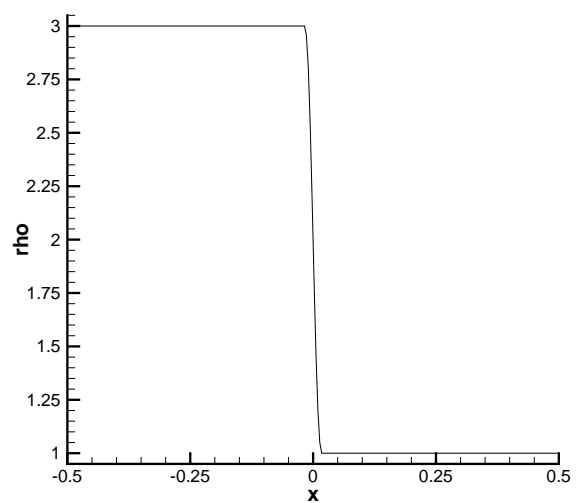

Fig. 5.7. $\rho$ component of the stationary contact problem at $t=0.25$, using second order HLL.

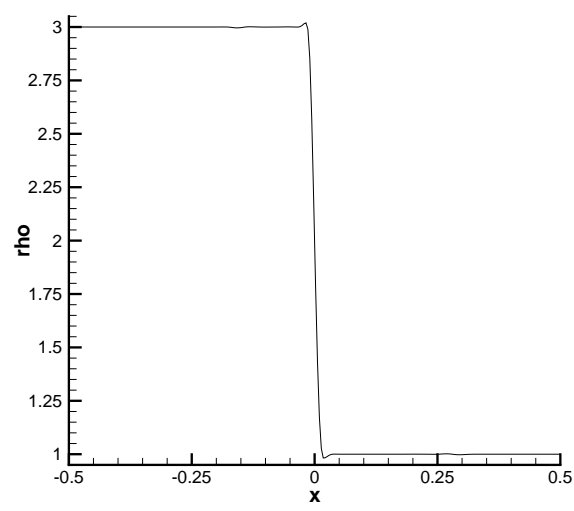

Fig. 5.8. $\rho$ component of the stationary contact problem at $t=0.25$, using second order MoT-ICE.

step, although the initial perturbation in the $p$ component is so small that it can hardly be seen in the plot (but it exists).

Anyway, we want to point out that the size of the emerged pressure waves is extremely small - don't forget to look at the scales, and for the $\rho$ component have a look at figure 5.8 for an unsplit plot.

The second and more important observation to mention is that MoT-ICE smears out the contact at least as much as HLL (remember that this is indicated by the width of the gap in the $\rho$ plot, or compare the unsplit plots in figures 5.7 (HLL) and 5.8 (MoT-ICE)). As pointed out in the introduction, this already suffices to demonstrate that the results of the MoT are not satisfactory in one spatial dimension. 
However, a single, one-dimensional test problem might be considered to be not a fair comparison, especially when comparing a Riemann solver based scheme to a scheme which was designed to capture multidimensional effects particularly well. Thus our next test problem was a stationary, planar contact oblique to a two-dimensional cartesian grid. In this situation, MoT-ICE again produced small oscillations and was about as dissipative as HLL. We do not display the figures, which look very similar as in the one-dimensional case.

Note that the small pressure waves generated - although they seem to be neglegibly small - can under certain circumstances lead to quite strange effects. For example, if a circular cloud is advected in a domain with periodic boundaries over many periods, the shape of the cloud can become very badly distorted. For details see Kröger [9]. These pressure perturbations also seem to be responsible for the distortions of radial symmetry in our next test case, see figure 5.9 below.

\subsection{Stationary disc}

Now we compute a disc at rest. The computational domain is $[-1,1] \times[-1,1]$, the radius of the disc is 0.5 . The density is 3 inside and 1 outside the disc, while velocity vanishes and the pressure is 1 everywhere. At the boundary of the computational domain, we used periodic boundary conditions. For cells lying on the boundary of the circular disc, we used an approximate weighted initial value.

We computed this problem up to $t=10$. Isoline plots are presented in figure 5.9. Again, we see that MoT-ICE is about equally dissipative as HLL. Moreover, MoT-ICE does not manage to preserve the circular symmetry, probably due to the small pressure perturbation observed in the previous example. Strong grid effects occur, whereas HLL gives an apparently perfect circle. The errors presented in table 5.1 verify that HLL's result is better than MoTICE's one: The absolute error of the HLL scheme is always smaller than that of MoT-ICE, and also, HLL has got a higher numerical order of convergence (about 0.8 , in the $L^{1}$ norm) than MoT-ICE (about 0.4). Furthermore, MoTICE increases the total variation considerably above the correct value (which is 8) whereas HLL does not seem to do so.

We also computed this example with zeroth order extrapolation at the boundary. The results look comparable to those with periodic boundary conditions.

Remarks 5.1 1) Note that in [11], the second author obtained perfectly radially symmetric shocks and rarefaction waves when applying the MoT-ICE to the shallow water equations. The present Euler solution is a stationary contact, a wave which is not contained in the shallow water equations. 

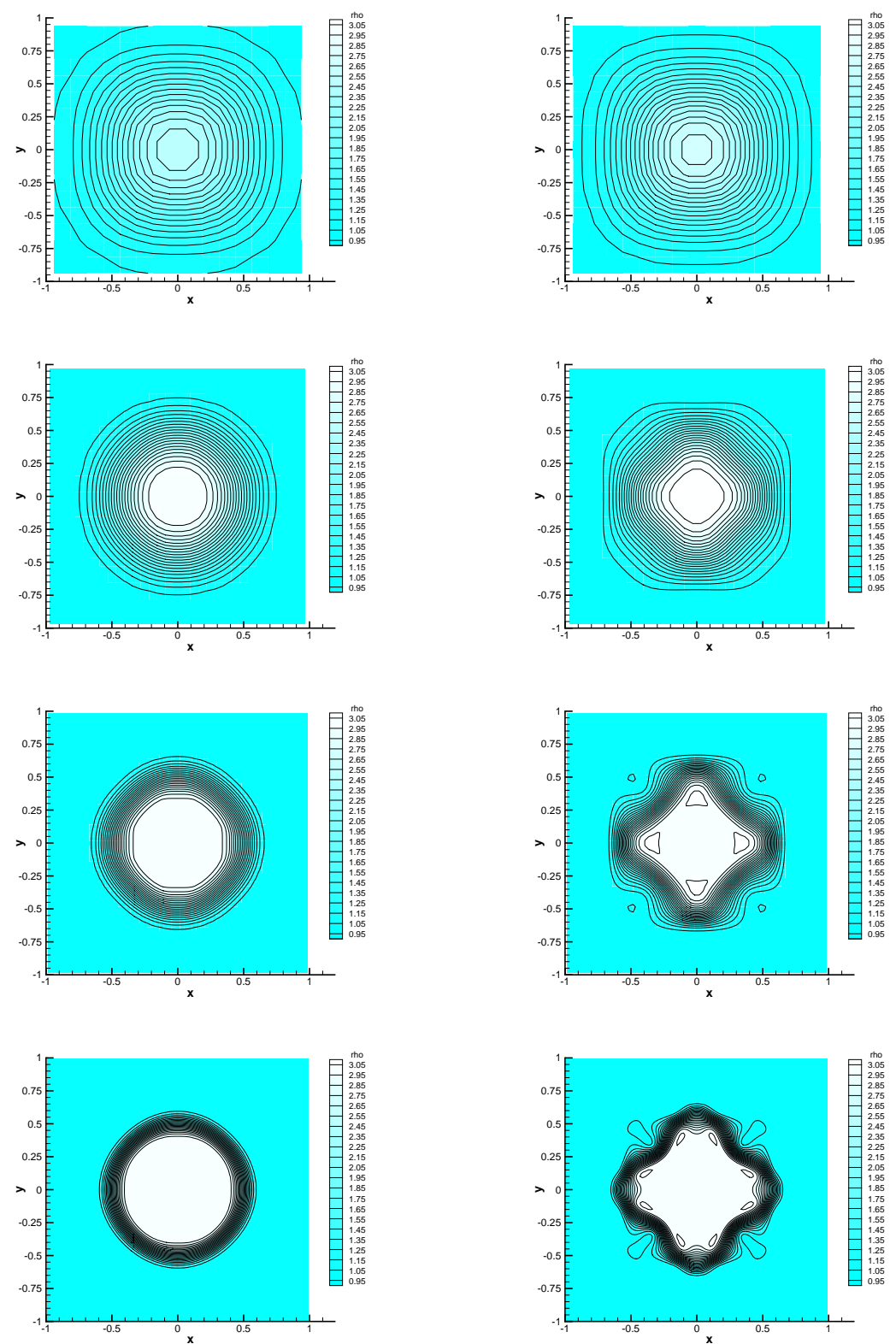

Fig. 5.9. Isoline density plots of the stationary disc problem. Isolines are seperated by 0.1 . Additionally, the plots are shaded with a light cyan color where the darkest value means $\rho=0.95$ and white means $\rho=3.05$. The left column shows HLL computations, the right column MoT-ICE computations; both were obtained with resolutions of $16 \times 16,32 \times 32,64 \times 64$ and $128 \times 128$ cells (from top to bottom). 
Table 5.1

$L^{1}$-errors (ERR), numerical order of convergence (NOC) and total variation (TV) in the $\rho$ component for the stationary disc problem. HLL (left), and MoT-ICE (right).

\begin{tabular}{r|ccccccc}
\hline & \multicolumn{3}{|c}{ HLL } & & \multicolumn{3}{c}{ MoT } \\
\cline { 2 - 5 } \cline { 5 - 7 } $1 / \Delta x$ & ERR & NOC & TV & ERR & NOC & TV \\
\hline 8 & $1.32+00$ & - & 5.89 & $1.19+00$ & - & 6.51 \\
16 & $7.10-01$ & 0.89 & 7.61 & $7.18-01$ & 0.73 & 8.13 \\
32 & $4.03-01$ & 0.82 & 7.93 & $5.12-01$ & 0.49 & 9.49 \\
64 & $2.26-01$ & 0.83 & 7.98 & $3.83-01$ & 0.42 & 9.83 \\
\hline
\end{tabular}

2) We conjecture that the main difficulty arising here is the continued production of small disturbances created by MoT-ICE at the contact discontinuities of the neighboring discs (in the case of periodic initial data, figure 5.9) or by reflections from the boundary (unless one uses perfectly nonreflecting boundary conditions).

\subsection{Oblique shear wave}

This is a Riemann problem with data oblique to the grid. The two states are connected by a shear wave only. The (unrotated) initial data is given by

$$
\begin{array}{lllll}
x<0: & \rho=1, & u^{x}=0, & u^{y}=1, & p=1, \\
x>0: & \rho=1, & u^{x}=0, & u^{y}=-2, & p=1,
\end{array}
$$

where $x$ ranges from -1 to 1 . The whole problem is then rotated by an angle of $\alpha=\arctan \left(s^{x} / s^{y}\right)$ anti-clockwise where $s^{x}$ and $s^{y}$ are positive integers. Then, the computation is taken place on a $512 \times s^{y}$ grid (where the ghost cell values at the north and south boundary are obtained from the opposite boundary, horizontally shifted by $s^{x}$ ) up to $t=0.5$, and finally the results are rotated back to obtain the plots.

For cells that lie on the discontinuity, area-weighted average values are used for the initial data. The averaging was performed in the primitive variables. However, averaging in the conservative variables yields no significant changes in the results. (Note that in our case the only difference is that for weighted conservative variables the pressure is not constant in the initial data although $p=1$ on both sides.)

We tested the following values for $s^{x} / s^{y}: 1 / 2,1 / 3,2 / 3,3 / 4$, and $4 / 5$. Results can be seen in figure 5.10. For this problem MoT-ICE gives better results than HLL (as usual, we compare the second order versions). Especially, HLL 

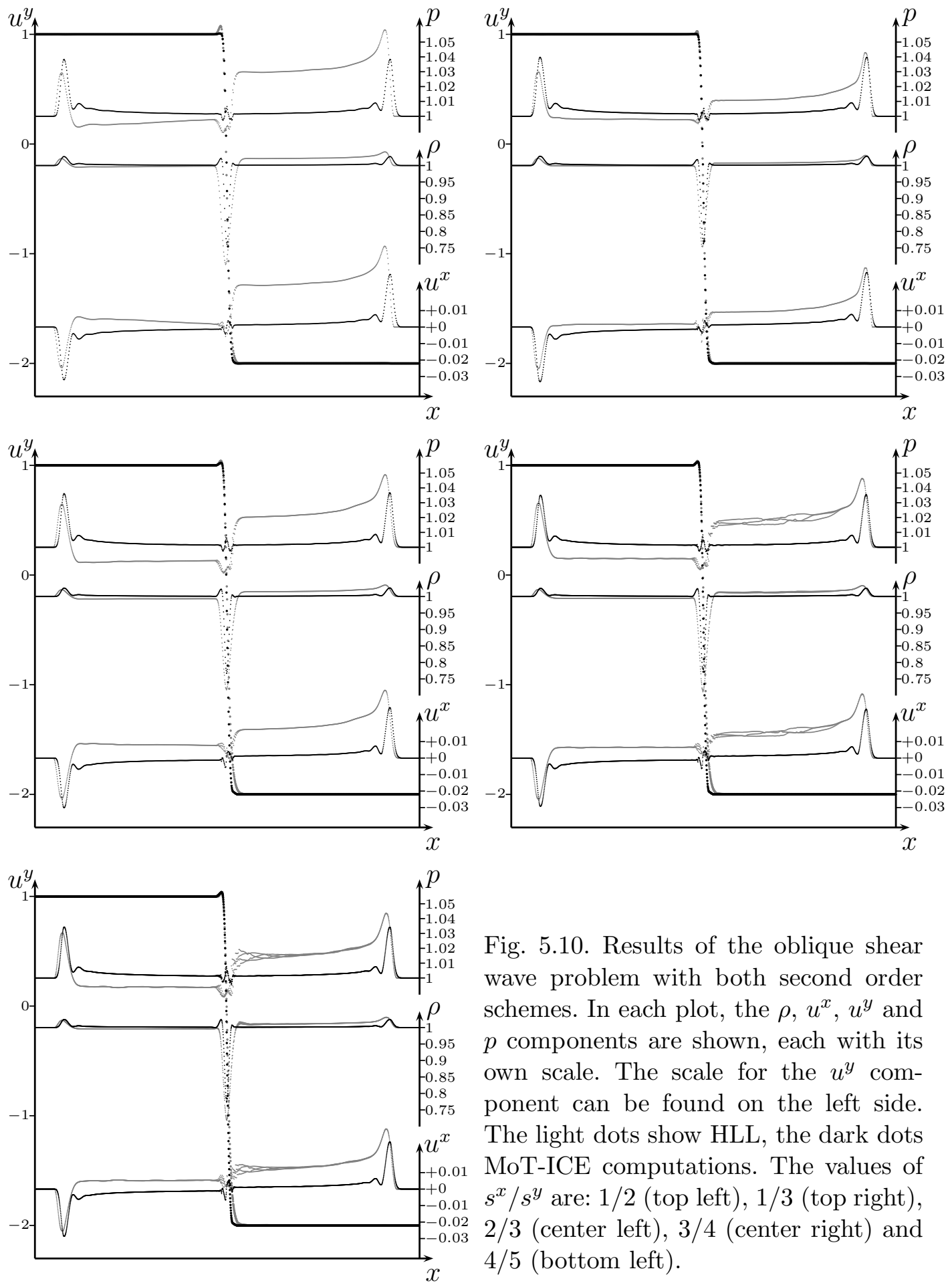

Fig. 5.10. Results of the oblique shear wave problem with both second order schemes. In each plot, the $\rho, u^{x}, u^{y}$ and $p$ components are shown, each with its own scale. The scale for the $u^{y}$ component can be found on the left side. The light dots show HLL, the dark dots MoT-ICE computations. The values of $s^{x} / s^{y}$ are: $1 / 2$ (top left), $1 / 3$ (top right), $2 / 3$ (center left), $3 / 4$ (center right) and $4 / 5$ (bottom left). 
produces wrong states on both sides of the shear wave in at least the $\rho, u^{x}$ and $p$ components. These wrong states depend strongly on the angle $\alpha$. For some values of $\alpha$, there are even some strange oscillations showing that there are different right states in each (rotated) $y$ layer. MoT-ICE does not produce such oscillations, gets the states on both sides of the shear discontinuity better, seems to be less sensitive to the angle $\alpha$ and is even significantly less dissipative than HLL.

It is interesting to perform a convergence test. In tables 5.2 and 5.3, this is done for the $\rho$ and $u^{y}$ components and $s^{x} / s^{y}=1 / 2$. We see that obviously MoT-ICE converges towards the correct solution at least with respect to $L^{1}$ norm, whereas HLL does not. This behaviour is shown in all components including the discontinuous $u^{y}$. (Values for $u^{x}$ and $p$ are not shown but behave similarly.)

We computed the same problem with either first order schemes, see figure 5.11 as well as tables 5.4 and 5.5 for the results. This time, both schemes get qualitatively correct results and $L^{1}$-converge towards the correct solution with approximately the same numerical order. MoT-ICE produces a slightly smaller absolute error than HLL at the same grid resolution, but still note that this is easily compensated by HLL being much faster.

Thus, HLL seems to be more sensitive to the effects of a second order reconstruction than the MoT-ICE. Note that we have reconstructed the solution dimension by dimension, and a more multidimensional reconstruction might cure the problem for HLL. In any case, we would like to point out that the results for this test problem should be interpreted with great care, since shear discontinuities are Kelvin-Helmholtz unstable [19].

\subsection{Strong stationary shocks}

This is a familiy of one-dimensional test problems introduced by Bultelle et al [1] (although they used it in a different context). The layout consists of a Riemann problem which is a stationary (but in general quite strong) shock. From the Rankine-Hugoniot condition, a straight forward computation shows that the general layout of a stationary shock is as follows:

$$
\begin{aligned}
& x<0: \quad \rho=\rho_{\mathrm{l}}, \quad \rho u=m_{\mathrm{l}}, \quad E=E_{\mathrm{l}}, \\
& x>0: \quad \rho=\rho_{1}(1+\xi), \quad \rho u=m_{1}, \quad E=E_{1}+\frac{1}{\gamma-1} \cdot \frac{3-\gamma}{2} \cdot \frac{m_{1}^{2}}{\rho_{1}}\left(1-\frac{1}{1+\xi}\right),
\end{aligned}
$$

where the following properties hold:

- The density $\rho_{\mathrm{l}}>0$ on the left side is a free parameter, 
Table 5.2

$L^{1}$-Errors (ERR) and numerical order of convergence (NOC) in the $\rho$ component for the oblique shear wave problem with $s^{x} / s^{y}=1 / 2$. HLL (left) and MoT-ICE (right).

\begin{tabular}{r|ccccc}
\hline & \multicolumn{2}{|c}{ HLL } & & \multicolumn{2}{c}{ MoT } \\
\cline { 6 - 6 } \cline { 5 - 5 } & ERR & NOC & ERR & NOC \\
\hline 32 & $7.84-02$ & - & $5.88-02$ & - \\
64 & $5.55-02$ & 0.50 & $3.69-02$ & 0.67 \\
128 & $4.15-02$ & 0.42 & $2.28-02$ & 0.70 \\
256 & $3.26-02$ & 0.35 & $1.40-02$ & 0.70 \\
512 & $2.72-02$ & 0.26 & $8.66-03$ & 0.69 \\
1024 & $2.46-02$ & 0.14 & $5.40-03$ & 0.68 \\
2048 & $2.39-02$ & 0.04 & $3.39-03$ & 0.67 \\
4096 & $2.60-02$ & -0.12 & $2.15-03$ & 0.66 \\
\hline
\end{tabular}

Table 5.3

$L^{1}$-Errors (ERR) and numerical order of convergence (NOC) in the $u^{y}$ component for the oblique shear wave problem with $s^{x} / s^{y}=1 / 2$. HLL (left) and MoT-ICE (right).

\begin{tabular}{r|cc|cc}
\hline & \multicolumn{2}{|c}{ HLL } & & \multicolumn{2}{c}{ MoT } \\
\cline { 2 - 3 } \cline { 5 - 5 } & ERR & NOC & ERR & NOC \\
\hline 32 & $1.96-01$ & - & $1.63-01$ & - \\
64 & $1.17-01$ & 0.75 & $9.49-02$ & 0.78 \\
128 & $7.07-02$ & 0.73 & $5.59-02$ & 0.77 \\
256 & $4.36-02$ & 0.70 & $3.31-02$ & 0.76 \\
512 & $2.67-02$ & 0.71 & $1.97-02$ & 0.75 \\
1024 & $1.65-02$ & 0.69 & $1.18-02$ & 0.74 \\
2048 & $1.15-02$ & 0.52 & $7.11-03$ & 0.73 \\
4096 & $1.06-02$ & 0.12 & $4.25-03$ & 0.74 \\
\hline
\end{tabular}



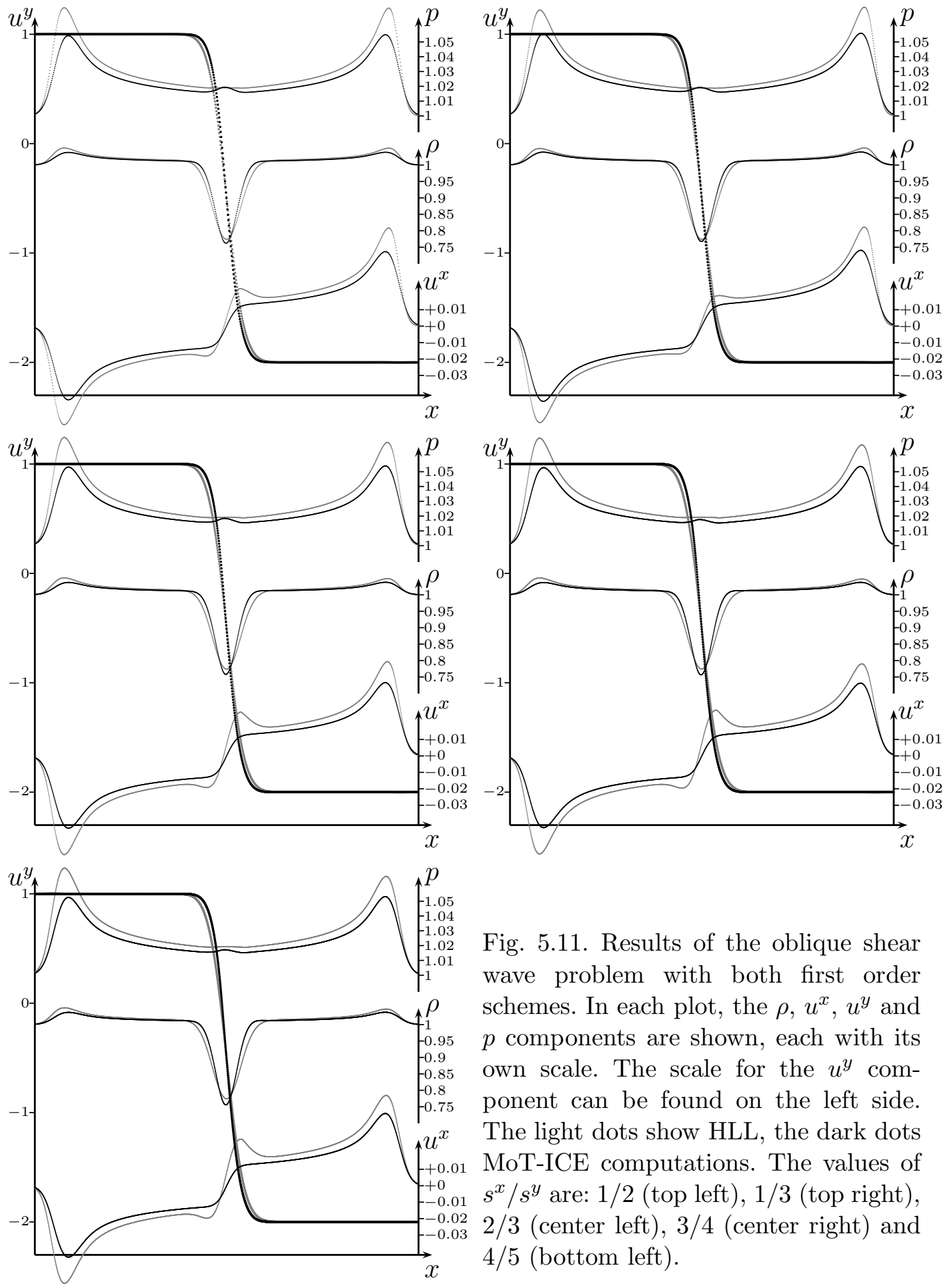

Fig. 5.11. Results of the oblique shear wave problem with both first order schemes. In each plot, the $\rho, u^{x}, u^{y}$ and $p$ components are shown, each with its own scale. The scale for the $u^{y}$ component can be found on the left side. The light dots show HLL, the dark dots MoT-ICE computations. The values of $s^{x} / s^{y}$ are: $1 / 2$ (top left), $1 / 3$ (top right), $2 / 3$ (center left), $3 / 4$ (center right) and $4 / 5$ (bottom left). 
Table 5.4

As table 5.2 ( $\rho$ component), but first order schemes.

\begin{tabular}{r|cc|cc}
\hline & \multicolumn{2}{|c}{ HLL } & & \multicolumn{2}{c}{ MoT } \\
\cline { 2 - 5 } \cline { 5 - 5 } & ERR & NOC & ERR & NOC \\
\hline 32 & $1.22-01$ & - & $1.10-01$ & - \\
64 & $1.14-01$ & 0.10 & $9.78-02$ & 0.18 \\
128 & $9.29-02$ & 0.30 & $7.83-02$ & 0.32 \\
256 & $7.12-02$ & 0.38 & $5.98-02$ & 0.39 \\
512 & $5.30-02$ & 0.43 & $4.45-02$ & 0.43 \\
1024 & $3.88-02$ & 0.45 & $3.26-02$ & 0.45 \\
2048 & $2.81-02$ & 0.46 & $2.36-02$ & 0.46 \\
4096 & $2.03-02$ & 0.47 & $1.70-02$ & 0.47 \\
\hline
\end{tabular}

Table 5.5

As table 5.3 ( $u^{y}$ component), but first order schemes.

\begin{tabular}{r|cc|cc}
\hline & \multicolumn{2}{|c}{ HLL } & & \multicolumn{2}{c}{ MoT } \\
\cline { 2 - 5 } \cline { 4 - 5 } & ERR & NOC & ERR & NOC \\
\hline 32 & $5.24-01$ & - & $4.16-01$ & - \\
64 & $3.67-01$ & 0.51 & $2.94-01$ & 0.50 \\
128 & $2.55-01$ & 0.53 & $2.07-01$ & 0.52 \\
256 & $1.77-01$ & 0.52 & $1.45-01$ & 0.51 \\
512 & $1.24-01$ & 0.52 & $1.01-01$ & 0.51 \\
1024 & $8.68-02$ & 0.51 & $7.17-02$ & 0.51 \\
2048 & $6.10-02$ & 0.51 & $5.05-02$ & 0.50 \\
4096 & $4.29-02$ & 0.51 & $3.56-02$ & 0.51 \\
\hline
\end{tabular}

- the momentum $m_{1}<0$ on the left side is a free parameter,

- energy $E$ and pressure $p$ are connected via the usual equation of state for an ideal gas:

$$
E=\frac{p}{\gamma-1}+\frac{\rho u^{2}}{2}
$$

- the energy $E_{1}$ on the left side is not a free parameter but given via the 
constraint that the pressure on the left side equals

$$
p_{1}=m_{1}^{2} \cdot \frac{\xi^{2}-\xi^{2} \gamma+2+3 \xi-\xi \gamma}{2 \gamma \rho_{1}(1+\xi)^{2}}
$$

- $-1<\xi<0$ is a free parameter, and

- the adiabatic coefficient $\gamma>1$ is also a free parameter.

In addition, note that the restrictions $m_{1}<0$ and $\xi<0$ are not really necessary, but if they are abandoned then in some cases left and right states will have to be interchanged because otherwise the entropy condition will no longer be satisfied. We will not study this in detail because we will always use examples where these restrictions are fulfilled.

Restricting ourselves even more, we only consider those three examples that were also considered by Bultelle et al. For all of these, we have $\rho_{1}=2$ and $m_{1}=-4$, and the remaining two free parameters are set to

$$
\begin{array}{ll}
\gamma=1.2, & \xi=-0.90825, \\
\gamma=1.65, & \xi=-0.75, \\
\gamma=3, & \xi=-0.4736 .
\end{array}
$$

They were computed with 512 cells on the interval $[-1,1]$ up to $t=0.2$ with both first and second order versions of either schemes. The results are presented in figure 5.12. For all cases, the momentum component is plotted. As Bultelle et al [1] indicated, this is a natural choice because this component is constant across a stationary shock.

The first observation is that in two of three cases, the second order MoT-ICE fails on the computations due to unphysical states. We recall that, following Zimmermann $[27,26]$, it should be possible to find conditions for the slope limiter which guarantee that the MoT-ICE does not produce unphysical states. However, this would require a transformation of each component of the state decomposition into a kind of 'pseudo-primitive' variables and then a reconstruction of these where the condition for the limiter is that each pseudodensity and each pseudo-pressure remain positive everywhere in the cell. We have not implemented this approach here.

HLL, on the other hand, reproduces the exact solution for all cases, with both first and second orders. Note, however, that for this problem we had to install a small fix which sets the signal velocities to zero if they are of size of the machine accuracy (we chose $10^{-10}$ as a limit). Without this fix, the roundoff errors amplify from step to step resulting in visible oscillations, but the results are still better than those of MoT-ICE. 

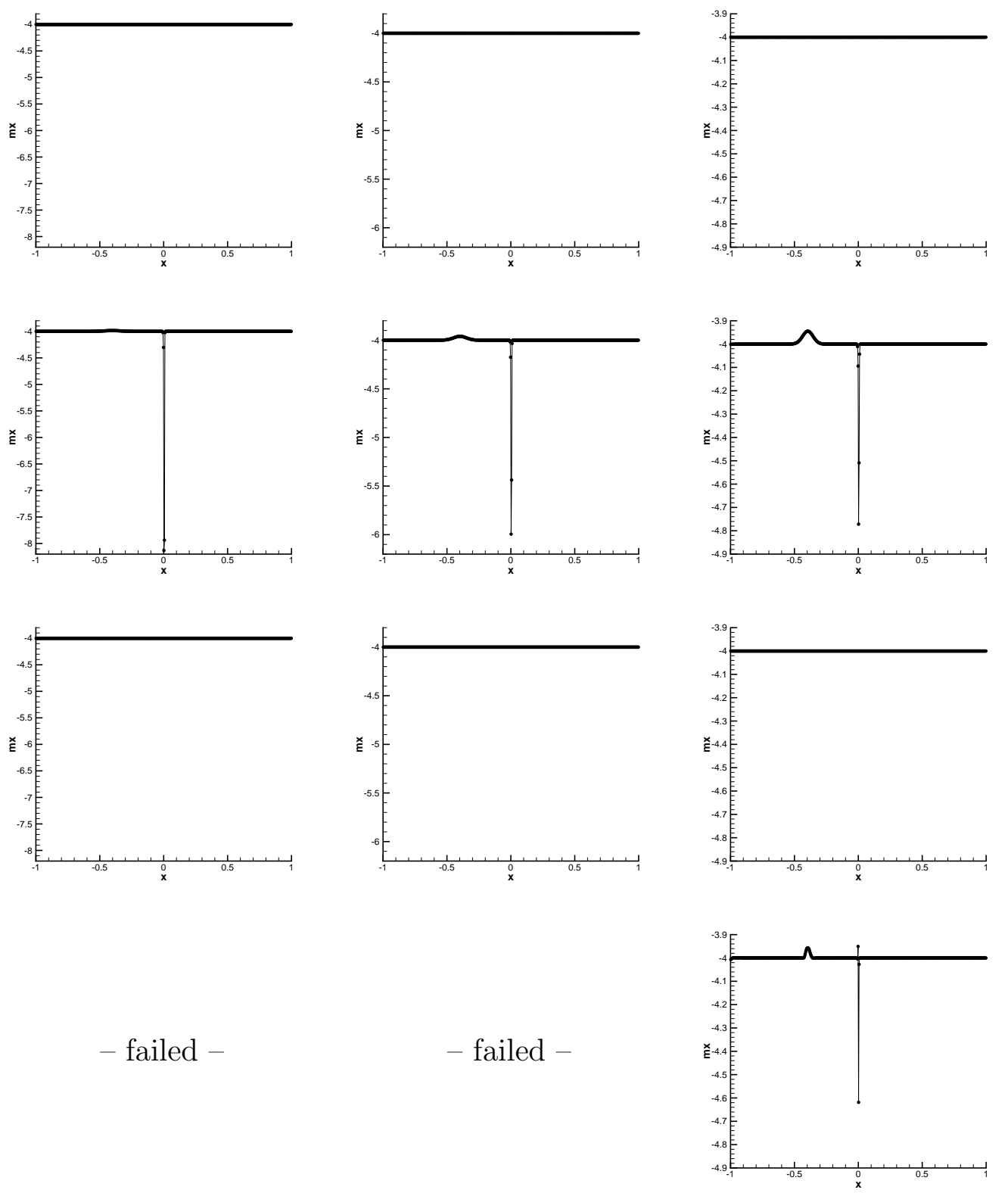

Fig. 5.12. The momentum component of the strong stationary shock. Left to right: Example problems nos. (5.1a), (5.1b), and (5.1c). Top to bottom: first order HLL, first order MoT-ICE, second order HLL, second order MoT-ICE. Missing plots are due to failing computations. 


\subsection{Shock entropy wave interaction}

This test problem was introduced by Shu and Osher [20]. It consists of a Mach 3 shock interacting with a sine wave in density. The inital data is as follows:

$$
\begin{array}{llll}
x<-4: & \rho=3.857143, & u=2.629369, & p=10.33333, \\
x \geq-4: & \rho=1+\varepsilon \sin 5 x, & u=0, & p=1 .
\end{array}
$$

The computational domain is $[-5,5]$ with zeroth order extrapolation at the boundary, and the sine wave amplitude $\varepsilon=0.2$. The computation is taken up to $t=1.8$. We computed the problem using HLL and MoT-ICE with 200 cells each and compared it to a reference solution obtained by HLL with 1600 cells. The reference solution does not change significantly if 3000 cells are used and apparently agrees with Shu's and Osher's reference solution.

The results are presented in figure 5.13. It can be seen in all components that MoT-ICE's resolution at the extrema of the sine wave is not significantly better than for HLL. The same applies for the resolution at the shock. MoTICE produces slightly inaccurate values at the right boundary. This might be due to the boundary conditions, although the same boundary conditions worked well for HLL.

For 400 cells (see figure 5.14), both schemes start to resolve the fine density waves near the $x$ interval $[1,2]$. Again, the quality of the results of both schemes is comparable.

\section{Conclusion}

We have compared the MoT-ICE (a multidimensional flux-vector-splitting scheme) to the classical HLL scheme for various test problems. For each scheme, we include first and second order versions. The main observations in favor of HLL are:

- MoT-ICE produces visible pressure oscillations at contacts, and these perturbations may seriously affect the symmetry of radial solutions with periodic boundary conditions. For HLL, we have proven that both first and second order versions keep velocity and pressure perfectly constant.

- MoT-ICE smears contact discontinuities (except shear waves) at least as much as HLL.

- The second order MoT-ICE failed for some strong stationary shocks.

- HLL computes radially symmetric solutions at least as well as MoT-ICE. 

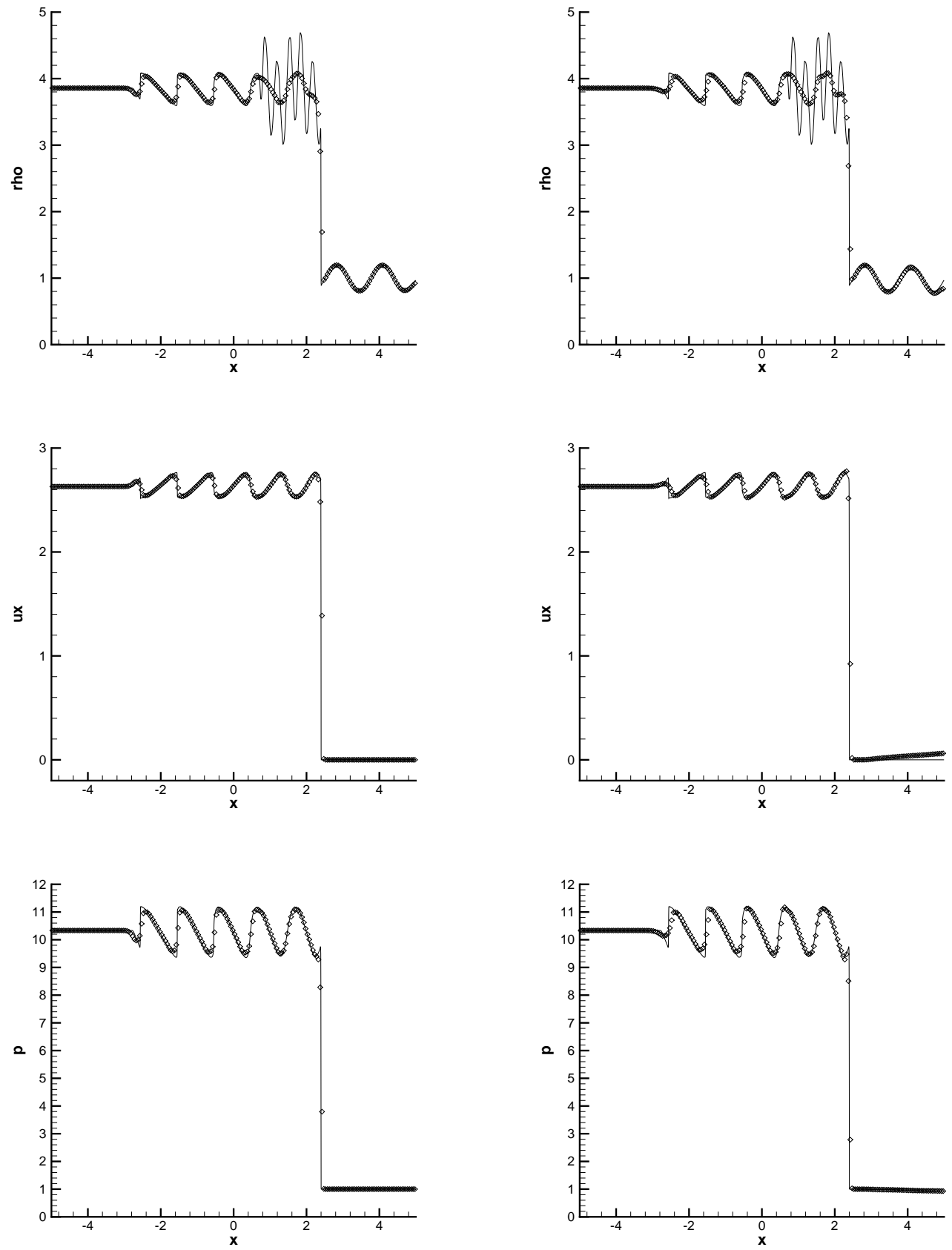

Fig. 5.13. Shock entropy wave interaction computed with 200 cells. Top to bottom: density, velocity, pressure. In every plot, the solid line is the reference solution and the diamonds are the solution obtained by HLL (left plots) or MoT-ICE (right plots). 

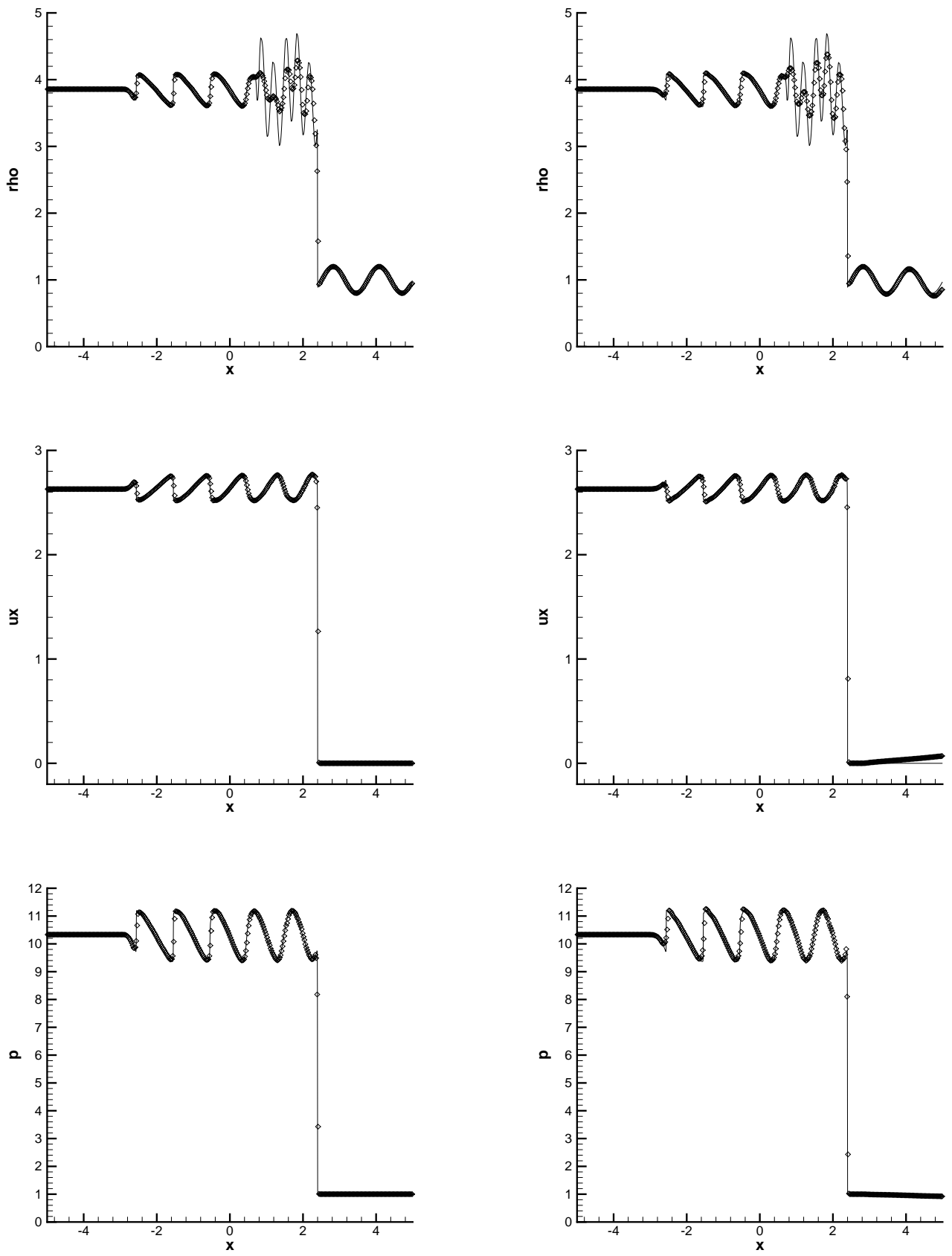

Fig. 5.14. Shock entropy wave interaction computed with 400 cells. Top to bottom: density, velocity, pressure. In every plot, the solid line is the reference solution and the diamonds are the solution obtained by HLL (left plots) or MoT-ICE (right plots). 
- HLL is significantly faster and easier to implement than MoT-ICE.

The main observation in favor of MoT-ICE is:

- For the oblique shear wave, the second order version of HLL did not converge to the correct solution, while MoT-ICE did. The first order versions of both schemes were roughly comprable.

If it were not for its failure on the oblique shear wave problem, HLL would be the clear frontrunner of our comparison. Thus, before further conclusions can be drawn, this problem must be investigated.

It would be interesting to see how other Riemann solver free schemes would perform for the test problems discussed herein.

Acknowledgement: The authors would like to thank Maja Lukáčová and Manuel Torrilhon for fruitful discussions.

\section{References}

[1] M. Bultelle, M. Grassin, D. Serre, Unstable Godunov discrete profiles for steady shock waves, SIAM J. Numer. Anal. 35 (1998), no. 6, 2272-2297.

[2] B. Einfeldt, On Godunov-type methods for gas dynamics, SIAM J. Numer. Anal. 25 (1988), no. 2, 294-318.

[3] B. Engquist, S. Osher, One-sided difference approximations for nonlinear conservation laws, Math. Comp. 36 (1981), no. 154, 321-351.

[4] M. Fey, Ein echt mehrdimensionales Verfahren zur Lösung der Eulergleichungen (Dissertation), Zürich (1993).

[5] M. Fey, Multidimensional upwinding. I. The method of transport for solving the Euler equations, J. Comput. Phys. 143 (1998), 159-180.

[6] M. Fey, Multidimensional upwinding. II. Decomposition of the Euler equations into advection equations, J. Comput. Phys. 143 (1998), 181-199.

[7] S. K. Godunov, A difference scheme for numerical computation of discontinous solutions of equations of fluid dynamics, Mat. Sb. 47, (1959), 271-306.

[8] A. Harten, P. D. Lax, B. van Leer, On upstream differencing and Godunov-type schemes for hyperbolic conservation laws, SIAM Rev. 25 (1983), no. 1, 35-61.

[9] T. Kröger, Dissertation, RWTH Aachen, Germany, in preparation.

[10] T. Kröger, S. Noelle, S. Zimmermann, On the Connection between some Riemann-Solver Free Approaches to the Approximation of Multi-Dimensional Systems of Hyperbolic Conservation Laws, IGPM Preprint no. 228, RWTH Aachen, Germany. Submitted to $M^{2} A N$. 
[11] S. Noelle, The MoT-ICE: A New High-Resolution Wave-Propagation Algorithm for Multidimensional Systems of Conservation Laws Based on Fey's Method of Transport, J. Comput. Phys. 164, (2000), no. 2, 283-334.

[12] S. Osher, F. Solomon, Upwind difference schemes for hyperbolic systems of conservation laws, Math. Comp. 38 (1982), no. 158, 339-374.

[13] M. Pandolfi, D. D'Ambrosio Numerical Instabilities in Upwind Methods: Analysis and Cures for the "Carbuncle" Phenomenon, J. Comp. Phys. 166 (2001), no. 2, 271-301.

[14] B. Perthame, Boltzmann Type Schemes for Gas Dynamics and the Entropy Property, SIAM J. Numer. Anal. 27, No. 6 (1990), 1405-1421.

[15] B. Perthame, Second-Order Boltzmann Schemes for Compressible Euler Equations in One and Two Space Dimensions, SIAM J. Numer. Anal. 29, No. 1 (1992), 1-19.

[16] J. J. Quirk, A contribution of the great Riemann solver debate, Int. J. Numer. Methods Fluids 18 (1994), 555-574.

[17] P. L. Roe, Approximate Riemann solvers, parameter vectors, and difference schemes, J. Comp. Phys. 43 (1981), no. 2, 357-372.

[18] P.L. Roe, Discrete models for the numerical analysis of time-dependent multidimensional gas dynamics, J. Comput. Phys. 63 (1986), 458-476.

[19] P. J. Schmid, D.S. Henningson, Stability and Transition in Shear Flows, Springer, New York (2001).

[20] C.-W. Shu, S. Osher, Efficient Implementation of Essentially Non-oscillatory Shock-Capturing Schemes, II, J. Comput. Phys. 83 (1989), no. 1, 32-78.

[21] P.K. Sweby, High resolution schemes using flux limiters for hyperbolic conservation laws, SIAM J. Num. Anal. 21 (1984), no. 2, 995-1011.

[22] C. v. Törne, MOTICE - Adaptive, Parallel Numerical Solution of Hyperbolic Conservation Laws (Dissertation), Bonner Mathematische Schriften, Nr. 334 (2000).

[23] E. F. Toro, Riemann solvers and numerical methods for fluid dynamics. A practical introduction, Second edition. Springer, Berlin (1999).

[24] E. F. Toro, M. Spruce, W. Speares, Restoration of the contact surface in the HLL-Riemann solver, Shock Waves 4 (1994), 25-34.

[25] G. Tóth, The $\nabla \cdot B=0$ Constraint in Shock-Capturing Magnetohydrodynamics Codes, J. Comput. Phys. 161 (2000), no. 2, 605-652.

[26] S. Zimmermann, The Method of Transport for the Euler Equations Written as a Kinetic Scheme, Internat. Ser. Numer. Math. 141 (2001), 999-1008.

[27] S. Zimmermann, Properties of the Method of Transport for the Euler Equations (Dissertation), Zürich (2001). 